\title{
EFFECT OF STOCHASTICS OF DIMENSIONAL PARAMETERS OF HAULING LAMINATED BUSHLESS CHAIN ELEMENTS OF IMPROVED STRUCTURE ON THEIR BEARING STRENGTH
}

\author{
Petro KRYVYI', Oleg LYASHUK'*, Petro KRYVINS'KYI', Maroš KORENKO² \\ 'Ternopil Ivan Pul'uj National Technical University, Ukraine \\ ${ }^{2}$ Slovak University of Agriculture in Nitra, Slovak Republic
}

\begin{abstract}
The distribution densities of dimensional parameters of a hauling laminated bushless chain of improved structure were investigated in this paper. The authors suggest calculating the non-uniform load of internal and external links plates by probability coefficient $K_{m^{\prime}}$ for which the minimum, the most probable and the maximum values were determined. The formula for $K_{m}$ determination depending on plate size variety due to a gap random value $\Delta$ and plate elastic deformation value under permitted payload was obtained. The effect of stochastics of dimensional parameters of hauling laminated bushless chain elements on their bearing strength is proved.
\end{abstract}

Keywords: hauling laminated chain, mathematical expectation, dispersion, bearing strength, permitted payload

According to Reshetov (1989), the bearing strength of chain gears equipped with power transmission bush and roller chains is determined (according to the basic criterion of their efficiency - chain hinges wear) for the case when pressure in hinges must not exceed the permitted pressure under given working conditions. Taking into account the fact given above, permitted actual force $F$ that can be passed by the chain with sliding joints equals to:

$$
F=\left[P_{0}\right] \times A / K_{e}
$$

where:

$p_{0}$ - permitted pressure in hinges for average operating conditions

A - supporting surface projection of the bush hinge $\left(\mathrm{mm}^{2}\right)$

$K_{e}$ - operation coefficient

Hauling laminated bushless chains (HLBC), which are widely used under easy working conditions in conveyers of various transporting machines, are calculated on bearing strength according to Ivashkov's method (Ivashkov, 1960).

The above-mentioned methods determining the bearing strength of power transmission bush and roller chains and HLBC do not take into account the stochastics of chain parts dimensional parameters such as centre-to-centre distance and diameters of plates holes, diameters of rollers and other, certainly influencing the inequality of pressures in chain hinges and tensions arisen in plates. It results in decreased chain bearing strength (Raskin, 1972), especially for the HLBC of improved structure, which is schematically shown in Figure 1.

Therefore, the theoretical research concerning the effect of stochastics of improved structure HLBC dimensional parameters on their bearing strength is an important problem.

\section{Material and methods}

The works of Ivashkov (1960), Hlushchenko (1953), Reshetov (1989), Serensen (1975), Kadnár (2011 and 2007) and others are devoted to the investigation of bearing strength, and the durability of machine hinged joints, including hinges of HLBC, is considered by Sukhariev (1977).

Investigating the stressed condition in chain hinges in operation (Ivashkov, 1960), it was accepted that hoop stress on a contour in the interval from $-\pi / 2$ to $\pi / 2$ (an angle of contact in the hinge is $2 \varphi_{0}=180^{\circ}$ ) is permanent and distributed uniformly, while according to Sukhariev (1977), the maximum angle of contact is $2 \varphi_{\max } \approx 85^{\circ}$ and stresses change according to the cosinusoidal law.

When calculating the operation coefficient, Reshetov (1989) states that it is necessary to take into account the stochastic nature of parameters it is determined by. According to Kryvyi et al. (1999), bearing strength was determined taking into account the stochastics of chains contact steps in parallel two-contour transmissions equipped with power transmission bush and roller chains.

The aim of work: determining the effect of stochastics of dimensional parameters of improved structure HLBC on their bearing strength.

Basic tasks:

1. To develop research methods dealing with the effect of stochastics of HLBC dimensional parameters on their bearing strength.

2. To determine the coefficient of plate non-uniform loading $K_{m}$ at transmission of HLBC under permissible hauling effort

3. To find the minimum, the most probable and the maximum values of $K_{m}$ and to determine their effect on the bearing strength of HLBC. 
Accepted assumptions:

1. Based on the central limit theorem and experimental data given in literature, we can accept that dispersion laws of such structural elements of HLBC as centre-tocentre distances of internal and external plates $-A_{v}$ and $A_{z}$ smaller than $d_{1}$ and larger than $d_{2}$ in diameter of plates holes and roller diameters $-d_{v}$ are normal.

2. The rigidity of HLBC plates at their tension is a constant value.

3. In the diagram of effort of plates extension deformation $\mathrm{F}-\Delta_{\mathrm{n} \mathrm{n}^{\prime}}$ index areas of contact rigidity are accepted as rectilineal.

4. The tense state of plates is examined in the area of elastic deformations only.

5. Mathematical expectations and mean values of hole diameters of internal and external plates, centre-to-centre distances of holes of internal and external plates, and the dispersion of given dimensional parameters are approximately equal, that is:

$$
\begin{aligned}
& M\left(d_{1 v}\right) \approx \bar{d}_{1 v}=M\left(d_{1 z}\right) \approx \bar{d}_{1 z} ; M\left(d_{2 v}\right) \approx \bar{d}_{2 v}=M\left(d_{2 z}\right) \approx \bar{d}_{2 z} ; \\
& M\left(A_{v}\right) \approx \bar{A}_{v}=M\left(A_{z}\right) \approx \bar{A}_{z^{\prime}} ;\left(d_{1}\right)=D\left(d_{2}\right) ; D\left(A_{v}\right)=D\left(A_{z}\right)
\end{aligned}
$$

where:

$M\left(d_{1 v}\right), M\left(d_{1 z}\right)$ and $\bar{d}_{1 z^{\prime}} \bar{d}_{1 v}-$ mathematical expectations and mean values of diameters of smaller holes of internal and external plates

$M\left(d_{2 v}\right), M\left(d_{2 z}\right)$ and $\bar{d}_{2 z^{\prime}} \bar{d}_{2 v}$ - mathematical expectations and mean values of diameters of larger holes of internal and external plates

$M\left(A_{v}\right), M\left(A_{z}\right)$ and $\bar{A}_{v}, \bar{A}_{z}$ - mathematical expectations and mean values of centre-to-centre distances of internal and external plate holes
$D\left(d_{1}\right)$ and $D\left(d_{2}\right)$ - dispersion of diameter of smaller and larger plate holes;

$D\left(A_{v}\right)$ and $D\left(A_{z}\right)$ - dispersion of centre-to-centre distances of internal and external plate holes

We use Figure 1 to determine the bearing strength of improved structure HLBC. Figure 1a schematically shows the structure of HLBC, and Figure 1b illustrates its dimensional parameters before and after permitted actual loading. The HLBC of improved structure (Figure 1a) consists of an internal link (1) connected by two parallel internal plates ( 2 and 3 ), where the holes $(4,6)$ of diameter $d_{1}$ are made. In these holes, the cylindrical end levels of the roller (5) are pressed into the internal plates holes $(7,8)$ of diameter $d_{2^{\prime}}$ where $d_{2}>d_{1}$, and the end levels of the roller (9) are loosely set (with a gap). As regards the external link (10) of HLBC, the cylindrical end levels of the roller (9) are pressed into the holes of diameter $d_{1}$ of the plates $(11,12)$, placed on both sides of the internal plates $(2,3)$, and the cylindrical end levels of the roller (15) are loosely made (with a gap) into the end levels (13 and 14) of diameter $d_{2}$ of the same plates. The instantaneous position of the HLBC segment is represented in Figure 1a where we can notice that the diameter of the hole (7) $d_{2, v,}$ of the left internal plate (2) is larger than the diameter of the hole (8) $d_{2 . z .}$ of the right internal plate (3), and the diameter of the hole (13) of the right external plate (11) $d_{2 . z . p}$ is smaller than the diameter of the hole (14) $d_{2 . z . l}$ of the left external plate (12).

In case of identical dimensional parameters of HLBC, using the permitted useful effort passed by the chain (Reshetov, 1989), its bearing strength is determined from the relationship:

$$
F_{i \delta}=2\left[p_{0}\right] \times A_{n} / K_{e}
$$

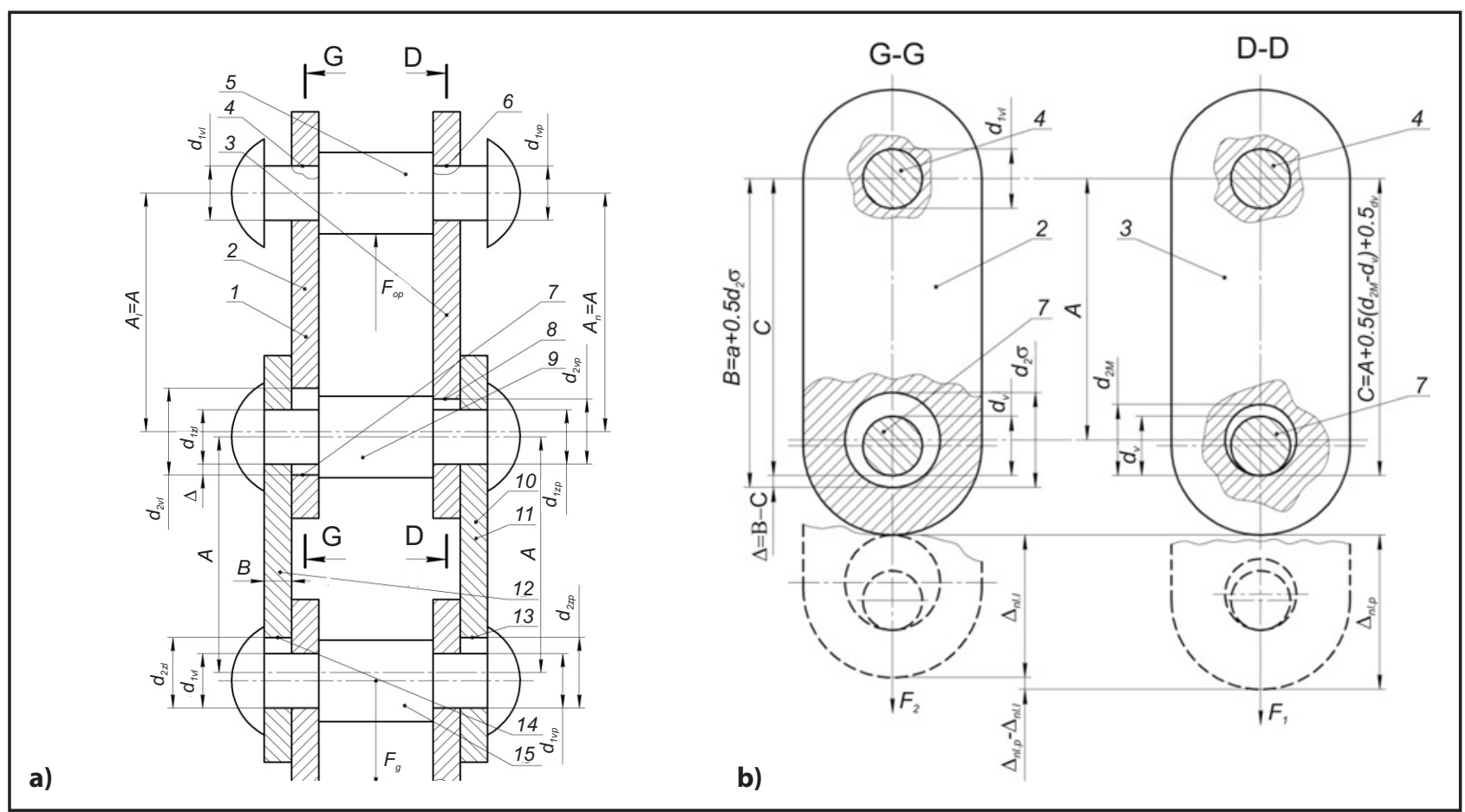

Figure 1 Structural scheme of improved structure HLBC (a) and its dimensional parameters before and after loading by permitted useful efforts (b) 
where:

$\left[p_{0}\right]$ - permitted pressure in hinges

$A_{n}$ - bearing surface area

$K_{e} \quad$ operation coefficient

As the dimensional parameters of HLBC (the diameters of rollers $d$ of the hole of smaller and larger diameters $d_{1}, d_{2}$ of plates) are stochastic values, then we use Figure 1 and Figure 2 to determine the permitted useful effort. To overcome the useful resistance force $F_{0}$ (Figure 1a), it is necessary to apply a certain effort $F>F_{0}$. Taking into account that the internal plates $(2,3)$ with the hole of diameter $d_{1}$ are pressed on the cylindrical end levels of the roller (5), and the external plates are connected in the same way with the roller (9), we will accept that the roller performs a flat parallel translation while applying the effort $F$. Then, if the gap $\Delta$ is present in the hinge, made by the hole (7) of greater diameter $d_{2}$ of the plate (2) and the diameter of cylindrical end levels $d$ of the roller (9) (Figure 1), only the plate (3) will be deformed at first. Then, choosing the gap $\Delta$, another internal plate (2) begins to deform. Having reached the loading of the right internal plate (3) by $F_{1}$ (Figure 2 ) and its elastic deformation $\Delta_{\text {nl. } p^{\prime}}$ the left internal plate will be deformed to the value $\Delta_{n ! l .}^{n ! p^{\prime}}=\Delta_{\text {nl.p }}+\Delta_{i}$ and will pass the loading $F_{2}$.

\section{Results and discussion}

In this case, the bearing strength of HLBC is permitted useful effort $F_{g}$, which will be determined from the relationship:

$$
F_{g}=\frac{K_{m}\left[p_{0}\right] \times A_{n}}{K_{e}}
$$

where:

$K_{m}$ - coefficient taking into account the non-uniformity of plate loading

According to Kryvyi et al. (1999), we have:

$$
K_{m}=2-\frac{\left|F_{1}-F_{2}\right|}{F_{1}+F_{2}}
$$

where:

$F_{1}$ and $F_{2}$ - loadings passed by the left (2) and right (3) internal plates (Figure 1). According to Equation (3), when $F_{1}=F_{2^{\prime}} K_{m}=2.0$ and the bearing strength of HLBC is equal to $F_{g}$. When $F_{1}=0$ or $F_{2}=0$, then $K_{m}=$ 1.0 and the chain can transmit actual load due to permitted pressure in the hinge of one plate

Expressing $F_{1}$ and $F_{2}$ in terms of the rigidity of plates and their deformations, when interplacing of plates holes is as represented in Figure 2, after simplification of Equation (3), we get:

$$
K_{m}=2-\frac{|\Delta|}{2 \Delta_{n l . p}-|\Delta|}
$$

where:

$2 \Delta_{n ! p}-$ (Figure 2) deformation of plate under its loading effort, which equals to $\left[p_{0}\right] \times d \times t$

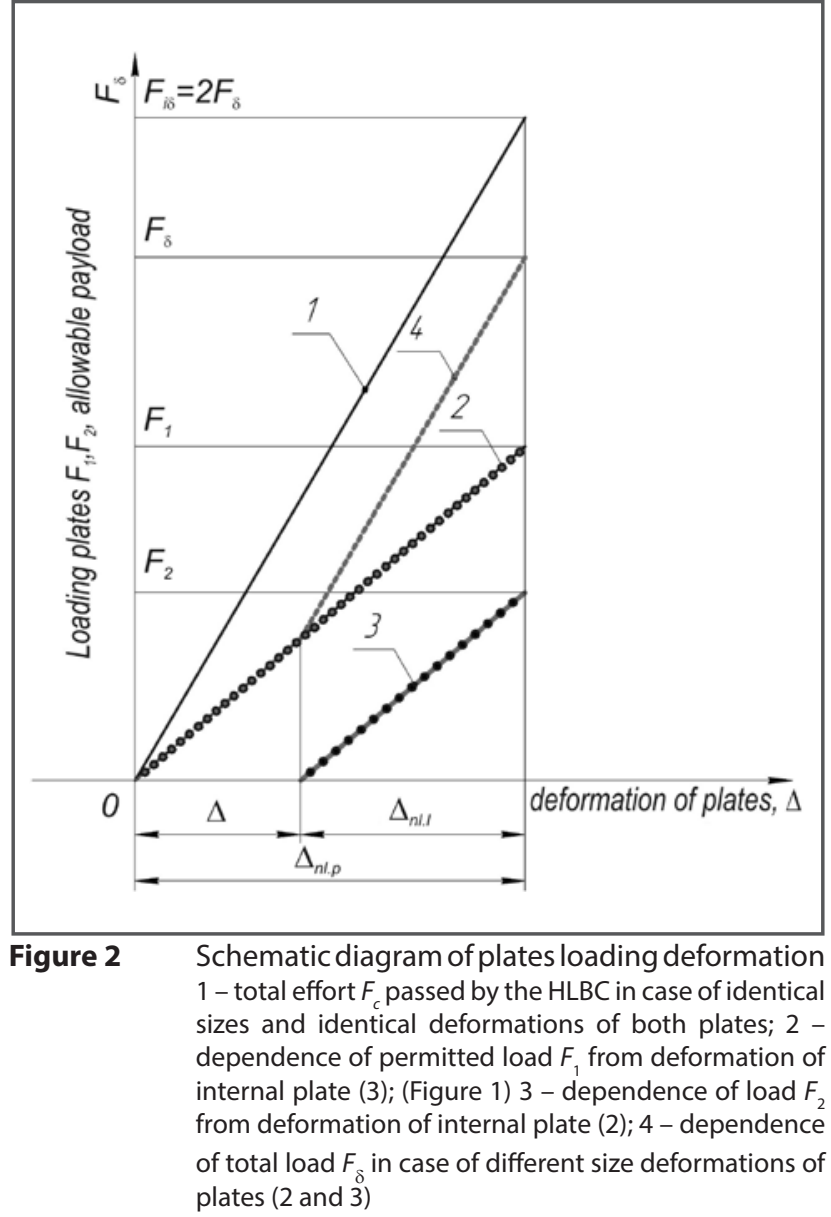

$t \quad-$ plate thickness

$\Delta>0$ - instantaneous value of gap $\Delta=d_{2 v, /}-d_{1 z . p}$ (Figure 1)

As the value $\Delta$ is formed by total dimensional parameters which are stochastic values, the value $\Delta$ will be a stochastic one, and taking into account Venttsel' (1969), we will accept hypothetically that it is submitted to the normal law of distribution.

Suppose that mean values of centre-to-centre distances of plates holes and areas of their dispersion are identicall for both external and internal plates, that is:

$$
\begin{gathered}
\bar{A}_{z}=\overline{A_{v}}=A_{n} \\
\sigma \sigma\left(A_{z}\right)=\sigma \sigma\left(A_{v}\right)=\delta
\end{gathered}
$$

where:

$A_{n}$ - nominal value of centre-to-centre distances of external and internal plates holes

$\sigma\left(\mathrm{A}_{z}\right)$ and $\sigma\left(A_{v}\right)$ - root-mean-square deviations of stochastic values $A_{z}$ and $A_{v}$

$\delta$ - corresponding field of admittance which is placed symmetrically on the value $A_{n}$

Based on the above-given facts and taking into account that $B=A+0.5 d_{2}$ (Figure $1 \mathrm{~b}$ ) as well as based on the normal law of distribution, the density distribution of values $B$ will be determined by the formula: 


$$
f(B)=\frac{12}{\sqrt{2 \pi\left(4 \delta_{A}^{2}+d_{2}^{2}\right)}} \exp \left\{-\frac{72\left[B_{i}-\left(A_{n}+0.5 \bar{d}_{2}\right)\right]^{2}}{4 \delta_{A}^{2}+\delta_{d_{2}}^{2}}\right\}
$$

The interval of the value $B$ is:

$$
A_{n}-0.5 \delta_{A}+0.5\left(d_{n}-\delta_{d 2 n}\right) \leq B \leq A_{n}+0.5 \delta_{A}+0.5\left(d_{n}+\delta_{d 2 v}\right)
$$

where:

$\delta_{d 2 v}$ and $\delta_{d 2 n}$ - upper and lower limits of diameter tolerances of plates larger holes

The mean value $\bar{B}$ of the value $B$ and dispersion $D(B)$ will be:

$$
\begin{gathered}
\bar{B}=A+0.5 \overline{d_{2}} \\
D(B)=\frac{4 \delta_{A}^{2}+\delta_{d_{2}}^{2}}{144}
\end{gathered}
$$

Using Figure $1 \mathrm{~b}$, the stochastic value $C$ is given in the form:

$$
C=A+0.5 d_{2 n p .}-0.5 d_{1}+0.5 d_{n p}
$$

where:

$d_{2 n p \text {. }}$ - diameter of the larger hole in the right plate; $d_{1}$ and $d_{2 n p}$ - diameters of the left and right ends of the roller

Due to the central limit theorem and taking into account that the members of the right side (Venttsel', 1969) are submitted to the normal law of distribution and the stochastic value $C$ is subordinated to the Gauss's law of distribution, the density of distribution $f(C)$ of the value $C$ is expressed as:

$$
\begin{gathered}
f(C)=\frac{12}{\sqrt{2 \pi\left(4 \delta_{A}^{2}+\delta_{d_{2}}^{2}+2 \delta_{d}^{2}\right)}} \exp \\
\exp \left\{-\frac{72\left[C_{i \delta}\left(A_{n}-0.5 \bar{d}\right)\right]^{2}}{4 \delta_{A}^{2}+\delta_{d_{2}}^{2}+2 \delta_{d}^{2}}\right\}
\end{gathered}
$$

where:

$\delta_{A^{\prime}} \delta_{d 2}$ and $\delta_{d}$ - tolerances of dimensions: centre-to-centre distance of plates holes; diameters of the larger plates holes and diameters of rollers ends

The characteristics of stochastic value $C$ dispersion according to the above-given pattern when determining in relation to the stochastic value $B$ are:

mean value:

$$
\bar{C}=A_{n}+0.5 \bar{d}_{2 n p}
$$

\section{dispersion:}

$$
D(C)=\frac{4 \delta_{A}^{2}+\delta_{d_{2}}^{2}+2 \delta_{d}^{2}}{144}
$$

Then, the density of distribution of the stochastic value $\Delta=B-C$, which is submitted to normal law, equals to:

$$
\begin{aligned}
f(\Delta)= & \frac{12}{\sqrt{2 \pi\left(8 \delta_{A}^{2}+2 \delta_{d_{2}}^{2}+2 \delta_{d}^{2}\right)}} \exp \\
& \exp \left\{-\frac{72 \Delta_{i}^{2}}{8 \delta_{A}^{2}+2 \delta_{d_{2}}^{2}+2 \delta_{d}^{2}}\right\}
\end{aligned}
$$

and its distribution characteristics are:

mean value:

$$
\bar{\Delta}=0
$$

\section{dispersion:}

$$
D(\Delta)=\frac{4 \delta_{A}^{2}+2 \delta_{d_{2}}^{2}+2 \delta_{d}^{2}}{144}
$$

We find the mathematical expectation $M(|\Delta|)$, variance $D(|\Delta|)$ and distribution density $f(|\Delta|)$ of a random variable of module $|\Delta|=|\mathrm{B}-\mathrm{C}|$ :

$$
M(|\Delta|)=\frac{\sqrt{2}}{2} \cdot \sigma(\Delta) \cdot e^{-\frac{\bar{\Delta}}{2 \sigma^{2}(\Delta)}}+2 \cdot \bar{\Delta} \cdot\left[\frac{\bar{\Delta}}{\sigma(\Delta)}\right]
$$

where:

$\Phi\left[\frac{\bar{\Delta}}{\sigma(\Delta)}\right]=\frac{1}{\sqrt{2 \pi}} \cdot \int_{0}^{\frac{\bar{\Delta}}{\sigma(\Delta)}} e^{-\frac{t^{2}}{2}} d t$ - value of Laplace function, $\bar{\Delta}$ and $\sigma(\Delta)$ average value and average quadratic deviation magnitude $\Delta$ :

$$
\begin{aligned}
& D(|\Delta|)=\Delta^{2}+\sigma^{2}(\Delta)- \\
& -\left[\sqrt{\frac{2}{\pi}} \cdot \sigma(\Delta) \cdot e^{-\frac{\Delta^{2}}{2 \sigma^{2}(\Delta)}}+2 \cdot \bar{\Delta} \cdot\left(\frac{\bar{\Delta}}{\sigma(\Delta)}\right)\right]^{2} \\
& f(|\Delta|)=\frac{12}{\sqrt{2 \pi\left(2 \delta_{A}^{2}+\delta_{d}^{2}\right)}} \exp \left\{-\frac{18\left|\Delta_{i}\right|^{2}}{2 \delta_{A}^{2}+\delta_{d}^{2}}\right\}
\end{aligned}
$$

$0 \leq|\Delta| \leq \delta_{A}^{2}+\delta_{d}^{2} ;|\bar{\Delta}|=0.133 \sqrt{\delta_{A}^{2}+\delta_{d}^{2}} ; \quad D|\Delta|=0.01 \sqrt{\delta_{A}^{2}+\delta_{d}^{2}}$

The theoretical distribution curves of the stochastic values $\Delta$ and $|\Delta|$ are given in Figure 3.

The minimum value of coefficient $K_{m}$ occurs when $|\Delta|$ is maximum, that is:

$$
K_{\text {min }}=2-\frac{3 \sigma(\Delta)}{2 \Delta_{i l}-3 \sigma(\Delta)}=2-\frac{0.5 \sqrt{\delta_{A}^{2}+\delta_{d}^{2}}}{2 \Delta_{i l}-0.5 \sqrt{\delta_{A}^{2}+\delta_{d}^{2}}}
$$

The maximum value of coefficient $K_{m}$ occurs when $|\Delta|=$ $=0$, that is $K_{m \text { max }}=2$.

The most probable value $K_{m . n . i .}$ is determined according to the following relation: 


$$
K_{\text {角.n. }}=2-\frac{0.133 \sqrt{\delta_{A}^{2}+\delta_{d}^{2}}}{2 \Delta_{i ̈ l}-0.133 \sqrt{\delta_{A}^{2}+\delta_{d}^{2}}}
$$

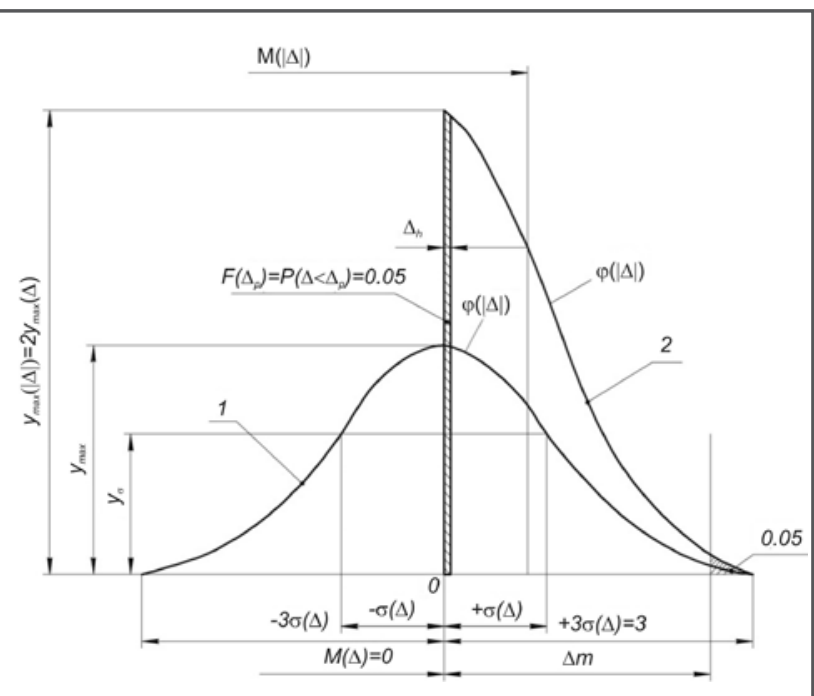

Figure 3 Theoretical distribution curves of stochastic values

1 - stochastic value $\Delta, 2$ - stochastic value $|\Delta|$

The estimation criterion of the effect of stochastics of HLBC dimensional parameters on bearing strength is assumed to be the minimum permitted value $K_{m . \delta^{\prime}}$ which is obtained at risk level 0.05 outside the top limit of the interval. To do this, it is necessary to find the quantile of the stochastic value $\Delta$, that is such a value $\Delta_{m}$ for which the function of distribution is $F\left(\Delta_{m}\right)=\mathrm{P}\left\{\Delta<\Delta_{m}\right\}=p=0.95$.

\section{Conclusions}

1. The theoretical research method for the effect of stochastics of HLBC elements parameters on bearing strength was offered, and relationships for determining the permitted useful effort that can be passed on the chain were obtained.

2. There were determined distribution densities of dimensional parameters of improved structure HLBC and analytical relationships to determine the coefficient $K_{m^{\prime}}$ taking into account the non-uniform loading of chain plates at certain values of gap $\Delta$ and elastic plates.
3. The relationships to determine the minimum, the maximum and the most probable value at a certain level of coefficient $K_{m}$ were obtained.

\section{References}

HLUSHCHENKO, I. P. 1953. About dependence of gesture of hauling laminated chain bone from its step. Scientific messages of LPCh, vol. 17, 1953, Mechanical series, sb. 4 .

IVASHKOV, I. I. 1960. Laminated chains. Constructing and calculation. Moscow : State Publishing. 1960, 264 pp.

KADNÁR, M. et al. 2011. The design and verification of experimental machine for real journal bearng testing. In Technički vjestnik, vol. 18, 2011, no. 1, p. 95-98. ISSN 1330-3651.

KADNÁR, M. - RUSNÁK, J. - KUČERA, M. 2007. Analýza kritických frekvencií pri skúškach na skúšobnom stroji Tribotestor. In Acta technologica agriculturae, roč. 10,2007, č. 3-4, s. 66-69. ISSN 1335-2555.

KRYVYI, P. - DUBYNIAK, S. - KUTSEVYCH, A. 1999. Bearing strength of two-contour parallel-row chain-drives. In Announcer of the Ternopil State Technical University, vol. 4, 1999, no. 3, pp.102-108.

KRYVYI, P. - LYASHUK, O. - KRYVINS'KYI, P. et al. 2013. Patent no. 81033. Ukraine laminated towing sleeveless chain. 2013. Digest no. $12,6 \mathrm{pp}$.

RASKIN, R. M. - KRONHAUS, V. S. - KIN, J. B. 1972. Chain-drives of chisel settings. Moscow : Niedra. 1972, 168 pp.

RESHETOV, D. N. 1989. Details of machines (Textbook for students of machine building and mechanical specialities). Moscow : Engineer. 1989, 496 pp.

SERENSEN, S. V. - KOCHAIEV, V. P. - SHNEIDEROVYCH, R. I. 1975. Bearing strength and calculations of machine parts on durability. Moscow : Engineer. 1975, 488 pp.

SUKHARIEV, I. P. 1977. Durability of hinged joints of machines. Certificate manual. Moscow : Engineer. 1977, 168 pp.

VENTTSEL', Y. S. 1969. Probability theory. Moscow : Publishing «Science». 1969, 576 pp.

\section{Contact address:}

Oleg Lyashuk, Ternopil Ivan Pul'uj National Technical University, Faculty of Computer Technologies, Department of Automated Control of Technological Processes, Ruska str. 56, 46000 Ternopil, Ukraine, e-mail: oleg-lashyk@rambler.ru 